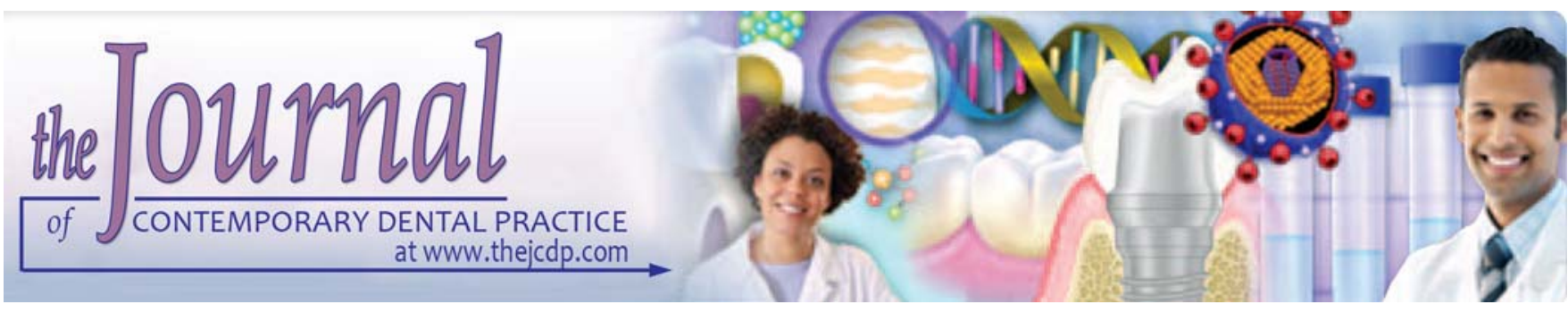

\title{
Finishing and Polishing Procedures of Composite Restorations by Saudi Dentists: A Cross-sectional Study
}

\author{
Mohammed Ali AlQarni, Rafi A Togoo, Ibrahim Al Shahrani, CR Sumanth Phani
}

\begin{abstract}
Aim: The purpose of this study was to assess the knowledge and practices of Saudi dentists in finishing and polishing composite restorations.
\end{abstract}

Materials and methods: A cross-sectional questionnaire-based study was conducted among 306 Saudi dentists from March to April 2012. The collected data were analyzed using Microsoft Excel 2010 program and descriptive statistics were obtained.

Results: Completed questionnaires were returned by 191 participants, giving a response rate of $62.4 \% .115$ (65.7\%) respondents are using fine diamond finishing burs for finishing occlusal surfaces, 82 (48\%) used fine aluminum oxide finishing discs for finishing facial surfaces, 130 (76\%) used diamond finishing strips for finishing interproximal surfaces.121 (63.4\%) respondents were of the view that the type of filler content has a major influence in providing color stability to the composite restoration and 137 (72.1\%) believed nano-composites provided enhanced color stability.

Conclusion: Dentists in Saudi Arabia are aware of the benefits of proper finishing and polishing of composite restorations. They however need to use finishing and polishing burs, disks, strips and pastes in a sequential series. Usage of composite polishing pastes and composite surface sealants should be emphasized in undergraduate curriculum.

Keywords: Composite restoration, Finishing, Dentists, Saudi Arabia.

How to cite this article: AlQarni MA, Togoo RA, Al Shahrani I, Phani CRS. Finishing and Polishing Procedures of Composite Restorations by Saudi Dentists: A Cross-sectional Study. J Contemp Dent Pract 2013;14(4):657-661.

Source of support: Nil

Conflict of interest: None declared

\section{INTRODUCTION}

Unesthetic teeth due to hypoplasia, fluorosis, fractures, caries and developmental disturbances can be treated using various esthetic techniques such as bleaching, esthetic contouring, composite restorations, porcelains and composite laminate veneering. Increasing demand for esthetic dentistry has been coupled with a rapid rate of development of new restorative resin-based materials. ${ }^{1}$

One of the most important steps in successfully creating bonded restorations is contouring, finishing and polishing. ${ }^{2}$ Proper finishing and polishing are important procedures that improve esthetics, maximize patients' oral health and increase the longevity of restoration. ${ }^{2,3}$ Staining or discoloration is one of the most common reasons for replacement of composite restorations ${ }^{4}$ and most of the published reports document that the finish, surface roughness and surface integrity of the restoration, as well as the physicochemical properties of the material itself can influence plaque adhesion and retention and staining of the composite resin.,

The dental practitioners' knowledge and attitudes toward the oral health care provide the frame work for their professional work. ${ }^{7}$ Dentists' treatment decisions are influenced by their knowledge toward care options, and assessing these is worthwhile. ${ }^{8}$ Composite resins like elsewhere are the most popular and commonly used esthetic restorative materials in Saudi Arabia and the key to beauty and biologic integrity of these long-term restorations lies in the final steps of the procedure. The aim of this study was to assess the knowledge and practices of general dentists and specialists in finishing and polishing composite restorations in Saudi Arabia.

\section{MATERIALS AND METHODS}

This cross-sectional questionnaire-based study of dentists was conducted from March to April 2012 in association with Saudi Dental Society. A prepiloted Questionnaire (Table 1) consisting of 17 questions was distributed among 306 dentists working in government and private dental clinics from ten geographically diverse regions of the country (Riyadh, Mecca, Madeena, Baha, Hail, Aseer, Jizan, 


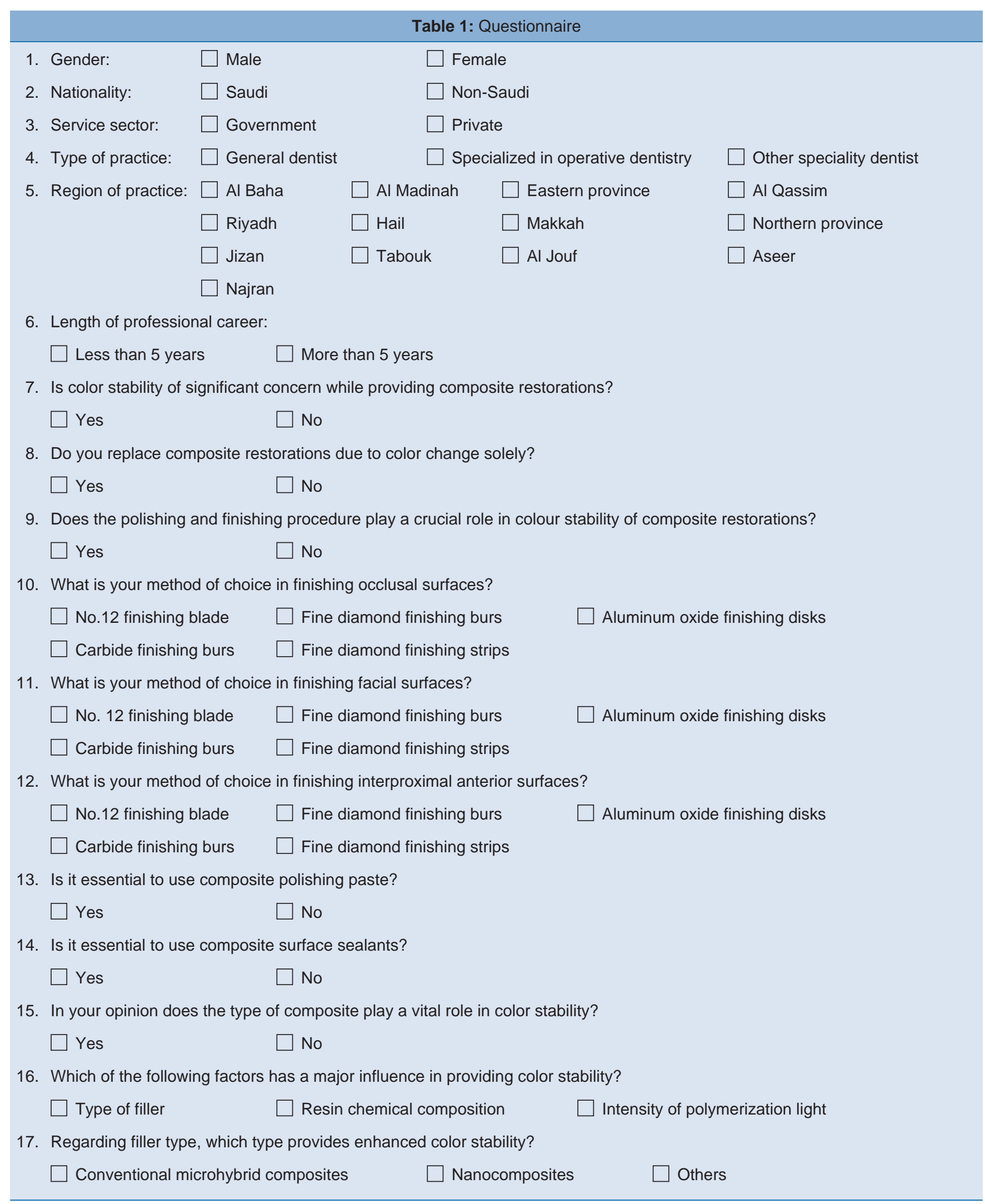

Najran, Eastern and Northern provinces) through electronic services of Saudi Dental Society research unit.

The sample was selected by simple random sampling from the Official Register of Saudi Dental Society and included general dentists as well as specialists. The participation of the dentists was voluntary and the electronic questionnaire was filled anonymously.

The participants were given a time frame of 4 weeks to reply and no attempt was made to send any reminder mails. The collected data were analyzed using Microsoft Excel 
2010 program and descriptive statistics were obtained. If the questionnaire was not filled completely, it was not excluded as a whole, but only the answered questions were taken into consideration in statistical analysis.

\section{RESULTS}

Completed questionnaires were returned by 191 participants, giving a response rate of $62.4 \% .99$ (51.8\%) were males and 92 (48.2\%) were females. The demographic data of the respondents can be summarized as follows - 126 (66\%) of the respondents were of Saudi nationality, the remaining 65 (34\%) were non-Saudi. 140 (74.1\%) worked in government sector and 49 (25.9\%) were from private sector. 80 (42.4\%) were general practitioners, 111 (57.6\%) were specialists . 120 (63.5\%) of them had more than 5 years of work experience and 69 (36.5\%) had less than 5 years. Dentists from all the ten geographically diverse regions in the country voluntarily participated in the study (Table 2).

The respondents expressed their opinions on color stability of composite restorations and usage of polishing paste and surface sealants (Table 3).

A total of 115 (65.7\%) respondents are using fine diamond finishing burs for finishing occlusal surfaces (Graph 1), 115 (48\%) are using fine aluminum oxide finishing disks for finishing facial surfaces (Graph 2), 130 (76\%) are using diamond finishing strips for finishing interproximal surfaces (Graph 3).

\section{Table 2: Region-wise participation of respondents}

\begin{tabular}{lc}
\hline Region & Respondents \\
\hline Al Baha & 3 \\
Al Madinah & 6 \\
Eastern province & 39 \\
Al Qassim & 1 \\
Riyadh & 86 \\
Hail & 2 \\
Makkah & 36 \\
Northern province & 2 \\
Jizan & 4 \\
Tabouk & 2 \\
Al Jouf & 1 \\
Aseer & 8 \\
Najran & 1 \\
\hline
\end{tabular}

When asked, which factor has a major influence in providing color stability to the composite restoration; 121 (63.4\%) respondents felt it to be type of filler content, 56 (29.3\%) of them felt it to be resin chemical composition and $14(7.3 \%)$ respondents felt it was intensity of polymerization light. When asked, which type of fillers provide enhanced colour stability; 137 (72.1\%) respondents felt it be nano-composites, 44 (23.2\%) of them felt it be conventional microhybrid composites and 9 (4.7\%) of them voted for other types.

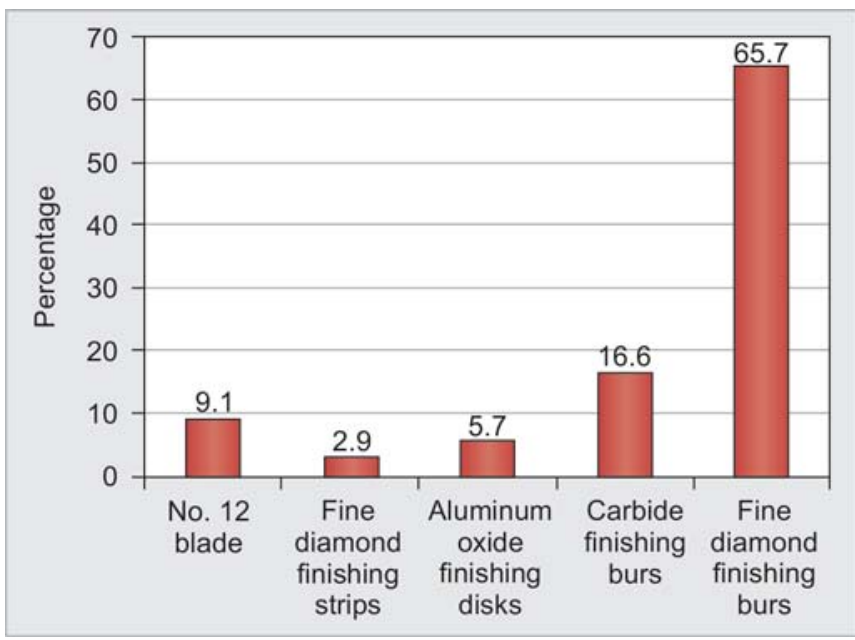

Graph 1: The practices of dentists in finishing occlusal surfaces

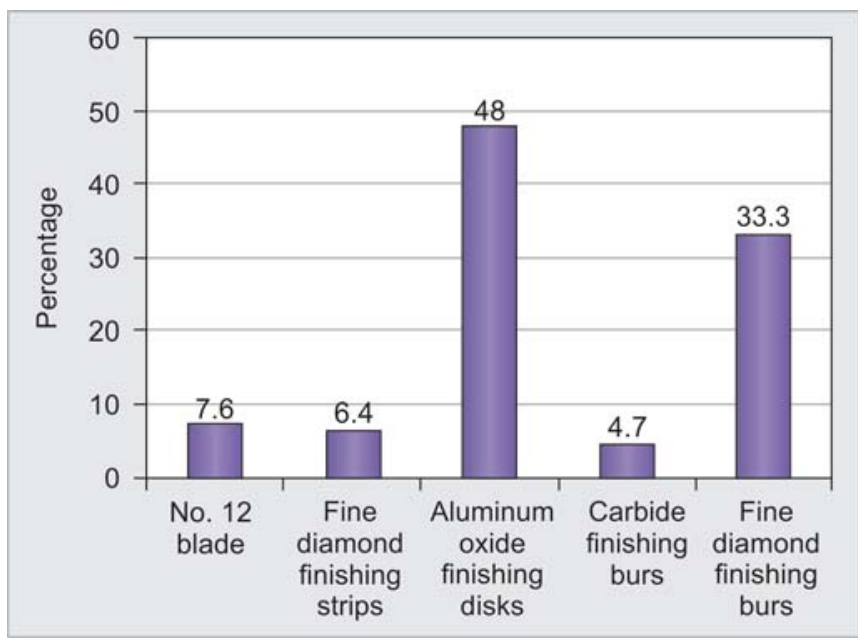

Graph 2: The practices of dentists in finishing occlusal surfaces

Table 3: Respondents' opinions on colour stability of composites

\begin{tabular}{|c|c|c|}
\hline Questions & Yes $n(\%)$ & No $n(\%)$ \\
\hline Is color stability of significant concern while providing composite restorations? & $174(91.6)$ & $16(8.4)$ \\
\hline Do you replace composite restorations due to color change solely? & $115(60.8)$ & $74(39.2)$ \\
\hline $\begin{array}{l}\text { Does the polishing and finishing procedure play a crucial role in color stability } \\
\text { of composite restorations? }\end{array}$ & $173(91.1)$ & $17(8.9)$ \\
\hline Is it essential to use composite polishing paste? & $65(37.4)$ & $109(62.6)$ \\
\hline Is it essential to use composite surface sealants? & $67(38.7)$ & $106(61.3)$ \\
\hline In your opinion, does the type of composite play a vital role in color stability? & $172(90.5)$ & $18(9.5)$ \\
\hline
\end{tabular}




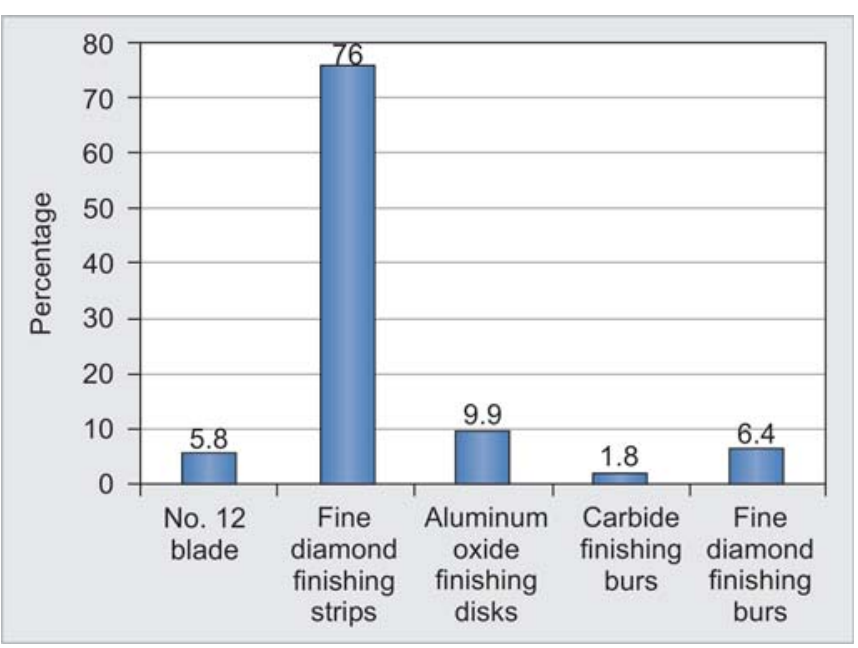

Graph 3: The practices of dentists in finishing interproximal surfaces

\section{DISCUSSION}

This is the first national study among the dentists in the Kingdom of Saudi Arabia to assess the knowledge and practices of dentists in finishing and polishing composite restorations. In interpreting the findings of the present study, it is important to acknowledge the possible limitations. As this is a self-reporting study, we could not determine whether reported practices reflected actual clinical practices and the cross-sectional data that is presented does not allow investigation of potential links between level of knowledge and attitude, to the practices used by dentists. The sample size was not as large as desired and was limited to Saudi Dental Society members. Despite these limitations, our results have important implications, since this is the first national study and the findings provide valuable baseline data about the knowledge and practices of dentists in finishing and polishing composite restorations in the kingdom.

Proper contouring, finishing, and polishing of anterior restorations is a key component to the long-term success of bonded restorations and is important for several reasons ${ }^{2,9}$ (Table 4). Similarly, 173 (91.1\%) respondents felt that polishing and finishing procedures play a crucial role in color stability of composite restorations.

Color stability of the composite resin materials is related to type of the composite resin restorative materials and polishing procedures. ${ }^{4}$ In agreement with this, 172 (90.5\%) respondents expressed that the type of composite plays a vital role in color stability. A Dutch study concluded that, staining or discoloration was one of the primary reasons for replacement of composite restorations. ${ }^{6}$ Similarly, 174 (91.6\%) respondents claimed that color stability is of significant concern while providing composite restorations and 115 (60.8\%) respondents claimed that they replace composite restorations due to color change solely.
Table 4: Advantages of proper finishing and polishing

- Greatly enhance the longevity, durability, and long-term wear resistance of the restoration

- Polishing the interproximal surfaces will significantly lower patients' risk for secondary caries and periodontal disease

- A smooth tooth surface minimizes gingival irritation and surface discoloration

- A polished tooth is more biologically compatible with the gingival tissue, so the health of the gingival tissue is maintained

- A smooth surface reduces the likelihood of adhesion, which means plaque is less likely to accumulate on a polished surface

- A highly polished tooth surface increases the reflective and refractive index of the restoration to create more natural and esthetic smiles

- Enhances patient comfort and satisfaction

Discoloration of composite restorations is caused by intrinsic and extrinsic factors. The intrinsic factors involve the discoloration of the resin material itself, such as the alteration of the resin matrix and of the interface of matrix and fillers. Extrinsic factors include staining by adsorption or absorption of colorants as a result of contamination from food and beverages. ${ }^{10}$ Bayne and Taylor ${ }^{1}$ stated that increasing the filler contents of composite resins generally improve the physical, chemical and mechanical properties such as water absorption, color stability and wear resistance. Some of the published studies ${ }^{4,11}$ have stated that staining is directly related to the resin phase of composites, where as some other studies suggested that the filler content is an important factor influencing composite color stability. ${ }^{12,13}$ In the present study, When asked, which factor has a major influence in providing color stability to the composite restoration; 121 (63.4\%) respondents felt it to be type of filler content, 56 (29.3\%) of them felt it to be resin chemical composition and $14(7.3 \%)$ felt it was intensity of polymerization light. When asked, which type of fillers provide enhanced color stability; 137 (72.1\%) respondents felt it be nanocomposites, 44 (23.2\%) of them felt it be conventional microhybrid composites and 9 (4.7\%) of them voted for other types.

Color stability of the composite resin materials is related to the type of composite resin and polishing procedures. Manufacturers provide a variety of instruments to accomplish finishing and polishing, such as diamond rotary cutting instruments, carbide burs, abrasive-impregnated rubber cups and points, abrasive disks and polishing pastes. Different types of composites call for different polishing techniques and various studies have reported that dedicated polishing systems and procedures are needed to create the kind of surface smoothness that prevents early discoloration. ${ }^{14-16}$ A total of 115 (65.7\%) respondents are using fine diamond finishing burs for finishing occlusal surfaces (Graph 1), 115 (48\%) are using fine aluminum 
oxide finishing disks for finishing facial surfaces (Graph 2), $130(76 \%)$ are using diamond finishing strips for finishing interproximal surfaces (Graph 3).

In the present study, 109 (62.6\%) and 106 (61.3\%) respondents felt it is not essential to use polishing paste and surface sealants (Table 3). Sen et $\mathrm{al}^{16}$ reported that the polishing pastes provided a smoother surface effectively and it has been reported that use of polishing paste after the use of polishing disks significantly decreased staining in comparison to the use of polishing disks alone, for all types of composites. ${ }^{4}$ Although long-term studies are not currently available to discuss the efficacy of composite surface sealants, short-term studies have shown that microcracks caused by the trauma of finishing procedures are resealed. ${ }^{17}$ Microcracks particularly at the cavosurface margins can propagate overtime, hence it is logical that the use of surface sealants postoperatively decrease the surface wear and increase the longevity of direct resin restorations. ${ }^{18}$

\section{CONCLUSION}

It is a positive finding to observe that dentists are aware of the benefits of proper finishing and polishing of composite restorations. Within the limitations of the present study, the following recommendations are put forth:

1. Need of continuing education programs and workshops to train the dentists in the use of a sequential series of finishing and polishing burs, disks, strips and pastes.

2. Usage of composite polishing pastes and composite surface sealants to be introduced at undergraduate level and emphasize on their importance.

\section{ACKNOWLEDGMENT}

The authors would like to acknowledge the cooperation of the Saudi Dental Society in the conduct of this survey.

\section{REFERENCES}

1. Bayne SC, Taylor DF, Sturdevant CM. Dental materials. The art and science of operative dentistry. 3rd ed. St Louis: Mosby 1995:206-287.

2. Mopper KW. Contouring, finishing, and polishing anterior composites. Inside Dentistry 2011;7:62-70.

3. Jefferies SR. The art and science of abrasive finishing and polishing in restorative dentistry. Dent Clin North Am 1998; 42:613-627.

4. Guler AU, Guler E, Yucel AC, Ertas E. Effects of polishing procedures on color stability of composite resins. J Appl Oral Sci 2009;17:108-112.

5. Bollen CML, Lambrechts P, Quirynen M. Comparison of surface roughness of oral hard materials to the threshold surface roughness for bacterial plaque retention: a review of the literature. Dent Mater 1997;13:258-269.
6. Kroeze HJP, Plasschert AJ, Van’t Hof MA, Truin GJ. Prevalence and need for replacement of amalgam and composite restorations in Dutch adults. J Dent Res 1990; 69:1270-1274.

7. Eijkman MAJ, De with C. Answers from dentists, dental hygienists and dental assistants to questions asked by patients concerning preventive dental matters. Community Dent Oral Epidemiol 1980;8:339-346.

8. McGlone P, Watt R, Sheiham A. Evidence-based dentistry: an overview of the challenges in changing professional practice. Br Dent J 2001;190:636-639.

9. Chung K. Effects of finishing and polishing procedures on the surface texture of resin composites. Dent Mater 1994;10:325-330.

10. Iazetti G, Burgess JO, Gardiner D, Ripps A. Color stability of fluoride containing restorative materials. Oper Dent 2000;25:520-525.

11. Reis AF, Giannini M, Lovadino JR, Ambrosano GM. Effects of various finishing systems on the surface roughness and staining susceptibility of packable composite resins. Dent Mater 2003;19:12-18.

12. Rodrigues SA Jr, Ferracane JL, Della Bona A. Flexural strength and Weibull analysis of a microhybrid and a nanofill composite evaluated by 3 - and 4-point bending tests. Dent Mater 2008;24:426-431.

13. Rodrigues SA, Scherrer SS, Ferracane JL, Della Bona A. Microstructural characterization and fracture behavior of a microhybrid and a nanofill composite. Dent Mater 2008; 24:1281-1288.

14. Reis AF, Giannini M, Lovadino JR, Santos Dias CT dos. The effect of six polishing systems on the surface roughness of two packable composite resins. Am J Dent 2002;15:193-197.

15. Roeder LB, Tate WH, Powers JM. Effect of finishing and polishing procedures on the surface roughness of packable composites. Oper Dent 2000;25:534-543.

16. Sen D, Goller G, Issever H. The effect of two polishing pastes on the surface roughness of bis-acryl composite and methacrylate-based resins. J Prosthet Dent 2002; 88:527-532.

17. Dickinson GL, Leinfelder KF. Assessing the long-term effect of a surface penetrating sealant. J Am Dent Assoc 1993;124:68-72.

18. Morgan M. Finishing and polishing of direct posterior resin restorations. Pract Proced Aesthet Dent 2004;16:211-216.

\section{ABOUT THE AUTHORS}

\section{Mohammed Ali AIQarni (Corresponding Author)}

Assistant Professor, Department of Restorative Dental Sciences, King Khalid University College of Dentistry, Abha, Saudi Arabia, Phone: +966-554155126, e-mail: dr.m.alqarni@hotmail.com

\section{Rafi A Togoo}

Associate Professor, Department of Preventive Dental Sciences, King Khalid University, College of Dentistry, Abha, Saudi Arabia

\section{Ibrahim Al Shahrani}

Assistant Professor, Department of Preventive Dental Sciences, King Khalid University, College of Dentistry, Abha, Saudi Arabia

\section{CR Sumanth Phani}

Assistant Professor, Department of Restorative Dental Sciences, King Khalid University, College of Dentistry, Abha, Saudi Arabia 